\title{
Documentos
}

\section{SALUD MATERNO-INFANTIL EN LAS AMÉRICAS}

\author{
Rogelio González P.
}

Departamento de Obstetricia y Ginecología, Facultad de Medicina, Pontificia Universidad Católica de Chile.

\section{RESUMEN}

Antecedentes: A nivel global, ocurren 136 millones de nacimientos, 10 millones de estos niños fallecerán antes de su primer año de vida. De la misma manera aproximadamente 500 mil madres morirán en el mismo período por causas asociadas al embarazo, parto y puerperio. El $99 \%$ de esta tragedia mundial ocurre en los países más pobres de la tierra. Más del $70 \%$ de las muertes maternas ocurren por cinco causas principales: hemorragias, infecciones, aborto, hipertensión y parto obstruido. Se ha observado un cambio en el perfil etiológico en aquellos países con experiencia mas favorables, como Cuba, Costa Rica, Uruguay y Chile, países en los cuales ha aumentado la proporción de las llamadas causas indirectas (no obstétricas) de mortalidad materna. La mortalidad infantil y la del menor de 5 años, ha disminuido en alrededor de un $50 \%$ en la región. Conclusión: A pesar de las mejorías globales en la salud materno-infantil y aumento de la esperanza de vida en la región de América Latina, la inequidad permanece como problema crucial. Existe información publicada que muestra que los mejores indicadores en salud materna, del recién nacido y del niño ocurre en mujeres con mejor situación socio-económica, estas diferencias se observan dentro y entre los países de la región. En el contexto del logro de los Objetivos Materno Infantiles del Milenio (ODM 4 y 5 ), es crucial la diseminación de las experiencias de los países con indicadores más favorables de la región.

\section{PALABRAS CLAVE: Mortalidad materna, mortalidad infantil, América Latina, inequidad, objetivos del milenio (ODM)}

\section{SUMMARY}

Background: One hundred and thirty six millions of births occurred annually at global level. Ten millions of those childrens will die before their first birthday. Approximately five hundred thousand of mothers also will die in the same period, for causes associated to pregnancy, delivery or puerperium. $99 \%$ of all of this global tragedy occurs in the poorest countries of the world. More than $70 \%$ of maternal death occurred because five main causes: hemorrhage, infections, abortion, hypertension and obstructed labor. A change in the etiological profile was observed, increasing the proportion of "indirect" (no obstetrics) causes in the countries with indicators more favorable as Cuba, Costa Rica, Uruguay and Chile. The infant and under five mortality in the region have been decreased in around 50\% between 1990 and 2005. Conclusion. Despite Latin America's overall declines in mortality and gains in life expectancy over the past few decades, inequity remains a leading health problem. Data from the region on maternal, newborn, infant, and child health show better outcomes among women with higher socioeconomic status in comparison with the poorest people within and also between the countries in the region. In the context of the global effort to attain Millennium Development Goals number 4 (reduce by two-thirds, between 1990 and 2015, the under-five mortality rate) 
and 5 (reduce by three-quarters, the maternal mortality ratio) and reduce health inequities, it is crucial to disseminate successful experiences in order that its achievements can be replicated elsewhere.

\section{KEY WORDS: Maternal mortality, infant mortality, Latin America, inequity, Millenium Developmental Goals (MDGs)}

\section{INTRODUCCIÓN}

A nivel mundial, la población se ha cuadruplicado en los últimos cien años, alcanzando actualmente a 6 mil millones de personas. Anualmente ocurren 136 millones de nacimientos, 10 millones de estos niños y niñas fallecen antes de cumplir 5 años y 8 millones antes del primer año de vida (WHO, 2005).

Aproximadamente 529.000 mujeres fallecen (razón de mortalidad materna 400/100.000 nacidos) en el mismo período, por causas asociadas al embarazo, parto o puerperio (WHO y cols., 2007). El $99 \%$ de estas muertes provienen del mundo en desarrollo, principalmente del sur de África y Asia y son evitables con cuidados médicos básicos y oportunos, asociados a sistemas de salud eficientes. La situación materno-infantil adquiere por lo tanto características de emergencia epidemiológica, con necesidad de intervenciones urgentes a nivel mundial (WHO, 2005).

Los indicadores de salud materno-infantil son considerados un reflejo del resultado de toda la situación de salud de un país. Representan el resultado de una sumatoria de factores económicos, educacionales, nutricionales y de acceso a redes de protección social.

Existe actualmente la voluntad política de las naciones por considerar la mortalidad materna y del niño como una emergencia global (OPS, 1991). Esto ha sido expresado por diversas organizaciones internacionales como las Naciones Unidas (NU), Organización Mundial de la Salud (OMS), Organización Panamericana de la Salud (OPS), UNICEF, Banco Mundial, como también por entidades creadas propiamente para estos fines, como la Alianza para la Salud de la Madre, del Recién Nacido y del Niño.

Actualmente existe consenso mundial sobre: el valor social y humano del embarazo, parto y puerperio, tanto para la madre y el recién nacido; la prioridad de alcanzar una atención profesional del parto para cada nacimiento; el reforzamiento del acceso y del funcionamiento de los sistemas de salud; el fortalecimiento de alianzas con la sociedad civil para el logro de una cobertura universal de la atención profesional para la madre y del recién nacido.

La superación de las llamadas tres "demoras": decisión de consultar, acceso a instalaciones de salud adecuadas y la obtención de una atención adecuada, incluyendo la derivación a niveles de mayor complejidad, siguen siendo etapas a superar, para alcanzar niveles óptimos de salud para la madre y el niño (Barnes-Josiah y cols, 1998).

Los indicadores de salud materna e infantil presentan una situación de mayor deterioro en el continente Africano y Asia, donde se concentran aproximadamente el $95 \%$ de las muertes maternas y perinatales (Tabla I) (WHO, 2006a).

\section{MORTALIDAD MATERNA: IMPACTO Y CAUSAS}

Más del $70 \%$ de las muertes maternas a nivel global son causadas por 5 causas principales: hemorragia, infecciones, aborto, hipertensión arterial y parto obstruido (WHO y cols, 2007). La hemorragia y la sepsis explican el 42 y $44 \%$ del total de los fallecimientos maternos en Asia y África y son evidentemente causas asociadas a insuficiente cobertura en la atención capacitada del parto. En una revisión sistemática realizada por OMS en 2006, aparece la hipertensión como primera causa de muerte materna para Latinoamérica y el Caribe (LAC), mientras que para los países desarrollados aparecen las llamadas "otras causas directas" y "causas indirectas", no propiamente obstétricas como de mayor repercusión en la letalidad; las causas asociadas a complicaciones del aborto y del parto obstruido explican el 12 y 13,4\% de las muertes maternas en LAC (Tabla II) (Khan y cols, 2006).

El cambio en el perfil etiológico hacia causas indirectas de mortalidad materna, ha sido también una situación observada para los países de LAC con los mayores progresos en sus indicadores de salud como son Cuba, Costa Rica, Uruguay y Chile (Donoso, 2004a; Donoso, 2004b; Donoso, 2006; Donoso \& Villarroel, 2003; Donoso \& Carvajal, 2008). 
Tabla I

MORTALIDAD MATERNA Y PERINATAL SEGÚN REGIONES DEL MUNDO (ESTIMACIONES OMS, 2000)

\begin{tabular}{|c|c|c|c|c|c|}
\hline Región & $\begin{array}{c}\text { Mortalidad } \\
\text { Materna } \\
\text { (muertes } \\
\text { por } 100000 \\
\text { nacidos } \\
\text { vivos) }\end{array}$ & $\begin{array}{c}\text { Número de } \\
\text { Muertes } \\
\text { Maternas }\end{array}$ & $\begin{array}{l}\text { Riesgo de } \\
\text { Mortalidad } \\
\text { Materna a lo } \\
\text { largo de la } \\
\text { vida }\end{array}$ & $\begin{array}{l}\text { Mortalidad } \\
\text { Perinatal } \\
\text { (muertes por } \\
1000 \\
\text { nacidos } \\
\text { vivos) }\end{array}$ & $\begin{array}{l}\text { Número de } \\
\text { Muertes } \\
\text { Perinatales }\end{array}$ \\
\hline África & 830 & 251.000 & $1: 20$ & 55,4 & 1.722 .000 \\
\hline Norte & 130 & 4.600 & $1: 210$ & 31,8 & 149.000 \\
\hline Sub-Sahara & 920 & 247.000 & $1: 16$ & 59,6 & 1.573 .000 \\
\hline Asia & 330 & 253.000 & $1: 94$ & 45,7 & 3.599 .000 \\
\hline Oriente & 55 & 11.000 & $1: 840$ & 32,1 & 666.000 \\
\hline Centro-Sur & 520 & 207.000 & $1: 46$ & 56,9 & 2.319 .000 \\
\hline Sur-Oriente & 210 & 25.000 & $1: 140$ & 34,8 & 417.000 \\
\hline Poniente & 190 & 9.800 & $1: 120$ & 36,7 & 196.000 \\
\hline \multicolumn{6}{|l|}{ América Latina } \\
\hline \& Países del Caribe & 190 & 22.000 & $1: 160$ & 21 & 244.000 \\
\hline Oceanía & 240 & 530 & $1: 83$ & 44,7 & 11.000 \\
\hline Total Mundial & 400 & 529.000 & $1: 74$ & 42,4 & 5.707 .000 \\
\hline Países Desarrollados & 20 & 2.500 & $1: 2800$ & 10,2 & 130.000 \\
\hline Países en Vias de Desarrollo & 440 & 527.000 & $1: 61$ & 45,8 & 5.577 .000 \\
\hline
\end{tabular}

Tabla II

CAUSAS DE MORTALIDAD MATERNA A NIVEL GLOBAL (ESTIMACIONES OMS, 192 PAÍSES: 1997-2002)(KHAN, 2006)

\begin{tabular}{lrrrr}
\hline & $\begin{array}{c}\text { Países } \\
\text { Desarrollados }\end{array}$ & África & Asia & $\begin{array}{r}\text { América Latina \& } \\
\text { Países del Caribe }\end{array}$ \\
\hline Número de Muertes Maternas & 2.823 & 4.508 & 11.777 \\
Hemorragia & $13,4 \%(4,7-34,6)$ & $33,9 \%(13,3-43,6)$ & $30,8 \%(5,9-48,5)$ & $20,8 \%(1,1-46,9)$ \\
Hipertensión & $16,1 \%(6,7-24,3)$ & $9,1 \%(3,9-21,9)$ & $9,1 \%(2,0-34,3)$ & $25,7 \%(7,9-52,4)$ \\
Sepsis/Infecciones & $2,1 \%(0,0-5,9)$ & $9,7 \%(6,3-12,6)$ & $11,6 \%(0,0-13,0)$ & $7,7 \%(0,0-15,1)$ \\
Abortos & $8,2 \%(0,0-48,6)$ & $3,9 \%(0,0-23,8)$ & $5,7 \%(0,0-13,0)$ & $12,0 \%(0,0-32,9)$ \\
Trabajo de Parto Obstruido & $0,0 \%(0,0-0,0)$ & $4,1 \%(0,0-10,3)$ & $9,4 \%(0,0-12,0)$ & $13,4 \%(0,0-38,9)$ \\
Anemia & $0,0 \%(0,0-0,0)$ & $3,7 \%(0,0-13,2)$ & $12,8 \%(0,0-17,3)$ & $0,1 \%(0,0-3,9)$ \\
HIV & $0,0 \%(0,0-0,0)$ & $6,2 \%(0,0-13,3)$ & $0,0 \%(0,0-0,0)$ & $0,0 \%(0,0-0,0)$ \\
Embarazo Ectópico & $4,9 \%(0,4-7,4)$ & $0,5 \%(0,0-3,3)$ & $0,1 \%(0,0-3,9)$ & $0,5 \%(0,0-4,5)$ \\
Embolismo & $14,9 \%(0,0-21,2)$ & $2,0 \%(0,0-5,6)$ & $0,4 \%(0,0-51,0)$ & $0,6 \%(0,0-8,4)$ \\
Otras Causas Directas & $21,3 \%(0,0-33,9)$ & $4,9 \%(0,0-10,3)$ & $1,6 \%(0,0-25,9)$ & $3,8 \%(0,0-27,9)$ \\
Otras Causas Indirectas & $14,4 \%(0,0-51,2)$ & $16,7 \%(9,1-29,3)$ & $12,5 \%(0,0-29,2)$ & $3,9 \%(0,0-25,3)$ \\
No Clasificables & $4,8 \%(0,0-22,9)$ & $5,4 \%(0,0-21,8)$ & $6,1 \%(0,0-16,2)$ & $11,7 \%(0,0-20,4)$ \\
\hline
\end{tabular}




\section{MORTALIDAD PERINATAL: IMPACTO Y CAUSAS}

Existe una fuerte asociación entre la morbimortalidad materna y perinatal. El $99 \%$ de 5,7 millones de muertes perinatales, ocurren también en el mundo en vías de desarrollo. Del total de muertes perinatales, 2,7 millones ocurren en la etapa antenatal (mortalidad fetal) y 3 millones en los primeros 7 días de vida (mortalidad neonatal precoz). Aproximadamente un tercio de la mortalidad perinatal en el mundo en desarrollo se asocia directa o indirectamente a eventos hipóxicos crónicos y agudos del parto: asfixia neonatal, insuficiencia placentaria y restricción del crecimiento intrauterino o el bajo peso al nacer. La prematurez esta asociada al $28 \%$ de las muertes neonatales, las anomalías congénitas e infecciones se asocian al resto de las causas de mortalidad neonatal (Lawn y cols, 2005). La mortalidad neonatal precoz representa el $38 \%$ de las muertes infantiles y el $29 \%$ del total de la mortalidad en menores de 5 años de vida (WHO, 2006a).

\section{MORTALIDAD INFANTIL: IMPACTO Y CAUSAS}

La estimación de muertes infantiles para el año 2000 fue de 10,8 millones de fallecimientos en menores de 1 año de vida. El $75 \%$ se concentra en África sub Sahara y el Sur de Asia. El 90\% se concentran en solo 42 países, considerados prioritarios para la Organización Mundial de la Salud.

En 1990 la mortalidad infantil bajo 5 años para estas regiones prioritarias, en relación al mundo industrializado fue de 180 vs $9 / 1000$ nacidos vivos (20 veces), respectivamente. La tendencia ha sido hacia el deterioro, en el 2000 esta diferencia aumentó de 175 vs $6 / 1000$ nacidos vivos (29 veces), respectivamente (Black y cols, 2003; Jiménez \& Romero, 2007).

Los eventos que ocurren en el periodo posneonatal e infantil constituyen el $64 \%$ de las causas de mortalidad bajo 5 años en el mundo en desarrollo. Una vez alcanzados niveles de salud adecuados en el niño y su entorno, la situación se invierte en los países con grados más avanzados de desarrollo, donde los eventos neonatales alcanzan al $52 \%$ de la mortalidad total hasta los 5 años. La OMS estima que a nivel global, el $32 \%$ de las causas de muerte bajo 5 años son atribuibles a diarrea y a neumonía y el $42 \%$ a causas neonatales (Mathers y cols, 2000; Black y cols, 2003).

La ocurrencia de las muertes neonatales, según las regiones del mundo, en relación a las muertes fetales y a las de todo el período infantil hasta los 5 años se muestra en la Tabla III (WHO, 2006b).

\section{OBJETIVOS DE DESARROLLO DEL MILENIO (ODM)}

Las naciones han definido metas a alcanzar para el año 2015, que incluyen el acceso universal a la educación primaria, la igualdad en derechos de géneros y empoderamiento de la mujer, el control de enfermedades como la malaria y el SIDA, el logro de un medio ambiente sustentable y alcanzar una maternidad segura para las mujeres y sus hijos. Además, se ha respaldado la proposición de que el mundo deba trabajar en alianzas de cooperación para lograr los objetivos propuestos (ONU, 2000). Los ODM 4 y 5 han sido definidos con objetivos específicos a alcanzar para los países al 2015: disminuir la mortalidad bajo 5 años de edad (ODM 4) y la mortalidad materna (ODM 5), en dos tercios (66\%) y tres cuartos $(75 \%)$, respectivamente, a partir de 1990. Aumentar la cobertura de vacunación del sarampión, de la atención profesional del parto y lograr acceso universal a la salud reproductiva para 2015, también son indicadores en el monitoreo de estos objetivos.

El llamado grupo de monitoreo global para el 2015 ("countdown 2015") ha reportado los progresos monitorizados en 68 países seleccionados. Seis de los 33 países más importantes de LAC forman parte de este monitoreo global para el 2015 (Bolivia, Brasil, Guatemala, Haití, Méjico, Perú). Para todos los ODM, existe una positiva tendencia en la región, excepto Haití, donde para el logro de estas metas, se necesita aún de un importante refuerzo de la cooperación internacional (Bryce \& Requejo, 2008). La mortalidad infantil bajo 5 años, considerado el principal indicador de salud infantil, ha mejorado en las regiones prioritarias en un $10 \%$ (185 a $166 \%$ ) y un $35 \%$ (126 a 82\%) en el Sur de África y de Asia, respectivamente, desde 1990 al 2005.

Para el 2005, la mortalidad materna a nivel global ha disminuido solo en un $5 \%$, desde 430 a 400 muertes por 100.000 nacidos vivos. Evaluaciones recientes, han demostrado que el avance en la cobertura de la asistencia del parto por personal capacitado ha sido insuficiente. Particularmente en el Sur de África y Asia donde el aumento ha sido de solo un 7 (42 a 45\%) y un $27 \%$ (30 a $38 \%$ ), desde 1990 hasta 2005, respectivamente. Esto ha sido reflejado en que las cifras de mortalidad materna en el área no se han modificado (Bryce y cols, 2008).

\section{SITUACIÓN REGIONAL EN LATINO-AMERICA Y EL CARIBE (LAC)}

A nivel general el continente americano enfrenta 
Tabla III

RELACIÓN ENTRE MORTALIDAD NEONATAL Y DIFERENTES PERÍODOS DE LA
MORTALIDAD INFANTIL. REGIONES DEL MUNDO (ESTIMACIÓN OMS 2003)

\begin{tabular}{lcccc}
\hline & $\begin{array}{c}\text { Mortalidad Neonatal } \\
\text { como porcentaje de } \\
\text { la mortalidad bajo } \\
5 \text { años }\end{array}$ & $\begin{array}{c}\text { Mortalidad Neonatal } \\
\text { como porcentaje de } \\
\text { la mortalidad infantil }\end{array}$ & $\begin{array}{c}\text { Mortalidad Neonatal } \\
\text { Temprana como } \\
\text { porcentaje de la }\end{array}$ & $\begin{array}{c}\text { Mortalidad Neonatal } \\
\text { Temprana como } \\
\text { porcentaje de la } \\
\text { mortalidad neonatal }\end{array}$ \\
$\begin{array}{l}\text { Mundial } \\
\text { mortalidad perinatal }\end{array}$ \\
$\begin{array}{l}\text { Regiones Desarrolladas } \\
\left(^{*}\right)\end{array}$ & 56 & 53 & 75 & 47 \\
Regiones en Desarrollo & 36 & 63 & 79 & 37 \\
África & 26 & 53 & 75 & 48 \\
Asia & 45 & 42 & 75 & 48 \\
América Latina \& Países & 42 & 61 & 75 & 47 \\
del Caribe & 34 & 51 & 77 & 55 \\
Oceanía & & 47 & 73 & 45 \\
\hline
\end{tabular}

(*) Australia, Nueva Zelandia y Japón se incluyen en Regiones Desarrolladas

según la Organización Panamericana de la Salud (OPS) tres grandes desafíos demográficos: el aumento poblacional, el envejecimiento de la población y la progresiva emigración hacia los sitios urbanos, particularmente hacia las grandes ciudades de la región. La población urbana ha aumentado desde 69 a un 79\% desde el año 1980 al 2006. Desde el punto de vista sanitario aumentó la esperanza de vida y disminuyeron significativamente las muertes por enfermedades endémicas infecciosas y por causa perinatal (Tabla IV) (OPS, 2007).

Desde 1950, la población del continente se triplicó, alcanzando al 2006 aproximadamente a 900 millones de habitantes (CEPAL, 2003). Durante la última década, el crecimiento promedio fue de $1,2 \%$ anual. El porcentaje de población mayor de 60 años, es igual o menor al 10\%, aun en la mayoría de los países de la región: Bolivia, Colombia, Ecuador, El Salvador, Guatemala, Guyana, Haití, Honduras, Méjico, Nicaragua, Paraguay, Perú, Republica Dominicana, Surinam y Venezuela. La población adulta mayor representa a más del $20 \%$ en Puerto Rico y Uruguay, situación similar a las naciones de Norteamérica. El porcentaje de población infantil refleja esta realidad epidemiológica, alcanzando niveles de 36 a 42\% para los mismos países con menor población adulta mayor (Tabla V).

Hacia el año 2006 la tasa de fecundidad alcanzó un rango entre 2,8 - 4,2 hijos/mujer para Bolivia, Guatemala, Honduras, Nicaragua, Paraguay y Republica Dominicana. Las tasas de natalidad oscilaron entre 23,5 a 33,2/1000 habitantes para estos mismos países, mientras que Estados Unidos y
Canadá, países desarrollados de la región, presentaron tasa de fecundidad y de natalidad de 2,1-1,5 y 14-10,3 respectivamente (OPS, 2007).

En la región, la cobertura de población bajo algún método de planificación familiar es alrededor del $60 \%$, porcentajes menores se observan en Bolivia, Republica Dominicana, Guatemala, Haití, Honduras, Méjico, Paraguay y Venezuela (Naciones Unidas, 2004).

El balance regional en el crecimiento económico ha sido positivo desde el año 1990 al 2005, con tasas de crecimiento sostenido promedio de 1,4 a 6\% (Naciones Unidas, 2005). El 41\% (213 millones) de los habitantes de la región, permanecen bajo la línea de la pobreza, el 17\% (88 millones) en condiciones de pobreza extrema, es decir insuficiente para cubrir sus requerimientos nutricionales básicos (internacionalmente menos de un dólar americano al día por persona). Aún en Honduras, Nicaragua, Bolivia y Paraguay, permanecen con niveles de pobreza sobre el $50 \%$ de sus poblaciones. El ingreso per cápita promedio de la región hacia el año 2005 fue de aproximadamente US\$7000. Aún permanecen dos países de la región con ingresos per cápita iguales o menores a US\$3.000, y por otro lado solo 6 países presentan ingresos sobre US\$10.000, incluyendo Estados Unidos y Canadá.

Durante el 2003, se estima que la región de LAC, orienta la mayoría de su gasto publico (como porcentaje de ingreso bruto) en seguridad social $(7,1 \%)$, seguido por educación $(4,1 \%)$ y finalmente salud y vivienda con un 2,9 y $0,9 \%$ respectivamente. Argentina, Costa Rica, Cuba y Panamá presentan los 
Tabla IV

PRINCIPALES INDICADORES DE SALUD EN CONTINENTE AMERICANO. DESDE 1980 Y PROYECCIÓN HASTA 2010.

\begin{tabular}{lccc}
\hline & $1980-1985$ & $1990-1995$ & $2005-2010$ \\
\hline Esperanza de vida al nacer (años) & 68,8 & 71,1 & 74,9 \\
Tasa total de fecundidad (hijos por mujer) & 3,1 & 2,6 & 2,8 \\
Mortalidad infantil (tasa por 1000 nacidos vivos) & 37,8 & 22,5 & 16,5 \\
Población urbana (\%) & 68,6 & 72,8 & 79,1 \\
Indicador & $1980-1985$ & $1990-1995$ & $2005-2010$ \\
Mortalidad por enfermedades transmisibles (tasa por & 109 & 62,8 & 55,9 \\
100.000 habitantes) & & & \\
Mortalidad por enfermedades del aparato circulatorio & 280 & 256,2 & 229,2 \\
(por 100.000 habitantes) & & & \\
Indicador & $1980-1985$ & $1990-1995$ & $2005-2010$ \\
Tasa de alfabetización (\%) & 88 & 87,6 & 93,8 \\
Cobertura de la inmunizacion (\%): DTP3 & 45 & 76,8 & 93 \\
Cobertura de la inmunizacion (\%): Antisarampión & 48 & 82,5 & 93 \\
Acceso al agua potable (\%) & 76 & 80 & 93 \\
Acceso a servicios de saneamiento (\%) & 59 & 66 & 84 \\
Enfermeras por cada 100.000 habitantes & 23,1 & 37,9 & 30 \\
\hline
\end{tabular}

porcentajes más alto de inversión relativa en salud (sobre 4\%), mientras que Bolivia, República Dominicana, Ecuador, El Salvador, Guatemala, Paraguay, Perú, Trinidad y Tobago, y Venezuela, destinan menos del $2 \%$ de sus ingresos en el sector salud (CEPAL/ECLAC, 2007).

El nivel educacional aumentó significativamente en los habitantes de la región, es así que el porcentaje de personas que alcanza el nivel primario de instrucción aumentó desde un 86 al 95 \% en 2004.

\section{SITUACIÓN MATERNO-INFANTIL REGIONAL}

En el continente americano viven aproximadamente 910 millones de personas, el $63 \%$ en la región de Latinoamérica y el Caribe (572 millones). Los nacimientos anuales son aproximadamente 16 millones, de los cuales el $71 \%$ corresponde a Latinoamérica.

Permanece la región de LAC anualmente con aproximadamente 22.000 muertes maternas, 480.000 en la etapa perinatal, 360.000 infantiles y 150.000 entre 1 y 4 años. En su gran mayoría son evitables con una atención profesional del parto y con sistemas de salud mejor organizados y accesibles para la población.

La razón de mortalidad materna estimada por OMS/OPS para la región a comienzos de los no- venta fue de 190/100.000 nacidos vivos (WHO, 2008). Cifra desproporcionadamente alta en relación al $72 \%$, y en aumento, de la atención profesional del parto: esta situación refleja un problema de calidad y capacidad en la organización de los servicios de salud de la región.

En la región la causas mas frecuentes de muerte materna son: hemorragias $(20 \%)$, preeclampsia $(22 \%)$, otras complicaciones posparto (15\%), otras causas directas $(17 \%)$ y causas asociadas al aborto $(11 \%)$. Solo en el $15 \%$ de las ocasiones, la causa esta asociada a la presencia de enfermedades pre-existentes agravadas por el embarazo. Esta distribución de causalidad refleja su evitabilidad en más del $80 \%$ de los casos.

A pesar de la disminución en los indicadores de mortalidad, la inequidad permanece como uno de los problemas de impacto en salud más importantes de la región (Bahr \& Wehrhahn, 1993; Braveman \& Tarimo, 2002; Casas y cols, 2001; Victora y cols, 1992; Donoso, 2004b; Wagstaff y cols, 2004).

Los mejores resultados en la salud materno-infantil regionales se observan en los quintiles de menores ingresos. El enfrentamiento y la disminución de la brecha en salud para los quintiles extremos han tenido resultados positivos en algunos países de la región (Acosta y cols, 2000; Almeida-Filho y cols, 2003). El mayor acceso a niveles superiores 
Tabla V

PRINCIPALES INDICADORES DEMOGRÁFICOS CONTINENTE AMERICANO

\begin{tabular}{|c|c|c|c|c|c|c|c|c|c|c|}
\hline & $\begin{array}{l}\text { Población } \\
\text { (miles) }\end{array}$ & $\begin{array}{l}\text { Población } \\
\text { urbana(\%) }\end{array}$ & $\begin{array}{c}\text { Población } \\
\text { menor de } \\
15 \text { años de } \\
\text { edad (\%) }\end{array}$ & $\begin{array}{c}\text { Población } \\
\text { mayor de } \\
60 \text { años } \\
(\%)\end{array}$ & $\begin{array}{l}\text { Población } \\
\text { Tasa de } \\
\text { crecimiento } \\
\text { anual (\%) }\end{array}$ & $\begin{array}{c}\text { Tasa de } \\
\text { fecundidad } \\
\text { total (hijos/ } \\
\text { mujer) }\end{array}$ & $\begin{array}{c}\text { Nacimientos } \\
\text { anuales (1.000 } \\
n v)\end{array}$ & $\begin{array}{c}\text { Tasa cruda } \\
\text { de natalidad } \\
\text { (por } 1.000 \\
\text { hab) }\end{array}$ & $\begin{array}{c}\text { Tasa cruda } \\
\text { de mortalidad } \\
\text { (por } 1.000 \\
\text { hab) }\end{array}$ & $\begin{array}{c}\text { Esperanza de } \\
\text { vida al nacer } \\
\text { (años) }\end{array}$ \\
\hline Argentina & 39.531 & 90,4 & 25,3 & 16,2 & 1 & 2,3 & 696,4 & 17,5 & 7,7 & 75,3 \\
\hline Bahamas & 331 & 90,8 & 26,2 & 10,9 & 1,2 & 2 & 5,6 & 16,9 & 6,1 & 73,5 \\
\hline Bolivia & 9.525 & 65,1 & 36,9 & 7,5 & 1,8 & 3,5 & 262,7 & 27,3 & 7,6 & 65,6 \\
\hline Brasil & 191.791 & 85,2 & 27,1 & 10,5 & 1,3 & 2,2 & $3.697,80$ & 19,2 & 6,3 & 72,4 \\
\hline Canadá & 32.876 & 80,3 & 16,5 & 21,6 & 0,9 & 1,5 & 341,2 & 10,3 & 7,4 & 80,7 \\
\hline Chile & 16.635 & 88,2 & 23,4 & 13,8 & 1 & 1,9 & 251,4 & 15 & 5,4 & 78,6 \\
\hline Colombia & 46.156 & 73,3 & 29 & 9 & 1,3 & 2,2 & 869,5 & 18,7 & 5,5 & 72,9 \\
\hline Costa Rica & 4.468 & 62,8 & 26,9 & 10 & 1,5 & 2,1 & 79,9 & 17,8 & 4,1 & 78,8 \\
\hline Cuba & 11.268 & 75,2 & 17,9 & 18,7 & 0 & 1,5 & 116,3 & 10,3 & 7,6 & 78,3 \\
\hline Ecuador & 13.341 & 63,8 & 31,4 & 10 & 1,1 & 2,6 & 281,6 & 21 & 5,1 & 75 \\
\hline El Salvador & 6.857 & 60,4 & 32,9 & 9,1 & 1,4 & 2,7 & 157,6 & 22,8 & 5,9 & 71,9 \\
\hline E.E.U.U. & 305.826 & 81,4 & 19,8 & 20,1 & 1 & 2,1 & $4.297,80$ & 14 & 8,2 & 78,2 \\
\hline Guadalupe & 445 & 99,8 & 22,9 & 17 & 0,7 & 2,1 & 6,6 & 14,8 & 6,4 & 79,2 \\
\hline Guatemala & 13.354 & 48,1 & 42,2 & 7 & 2,5 & 4,2 & 450,3 & 33,2 & 5,7 & 70,3 \\
\hline Guyana & 738 & 28,3 & 30,4 & 10 & $-0,2$ & 2,3 & 12,5 & 17,1 & 8,4 & 66,8 \\
\hline Haití & 9.598 & 40,1 & 37 & 6,8 & 1,6 & 3,5 & 270,2 & 27,9 & 9,2 & 60,9 \\
\hline Honduras & 7.106 & 47,4 & 38,5 & 6,8 & 1,9 & 3,3 & 200,3 & 27,9 & 5,6 & 70,2 \\
\hline Jamaica & 2.714 & 53,7 & 30,3 & 11,9 & 0,5 & 2,4 & 54 & 19,9 & 7,1 & 72,6 \\
\hline Martinica & 399 & 98 & 20 & 20,4 & 0,3 & 1,9 & 4,9 & 12,4 & 7,6 & 79,5 \\
\hline México & 106.535 & 76,5 & 29,3 & 10 & 1,1 & 2,2 & $2.075,30$ & 19,3 & 4,8 & 76,2 \\
\hline Nicaragua & 5.603 & 59,8 & 36,2 & 6,7 & 1,3 & 2,8 & 140,4 & 24,9 & 4,7 & 72,9 \\
\hline Panamá & 3.343 & 72,4 & 29,4 & 10,3 & 1,6 & 2,6 & 70,2 & 20,8 & 5 & 75,5 \\
\hline Paraguay & 6.127 & 59,7 & 34,6 & 8,1 & 1,8 & 3,1 & 153,4 & 24,8 & 5,5 & 71,8 \\
\hline Perú & 27.903 & 73 & 30,4 & 9,3 & 1,2 & 2,5 & 586,1 & 20,9 & 6,1 & 71,4 \\
\hline Puerto Rico & 3.991 & 98,1 & 20,6 & 20,6 & 0,5 & 1,8 & 53,4 & 13,3 & 7,6 & 78,7 \\
\hline $\begin{array}{l}\text { República } \\
\text { Dominicana }\end{array}$ & 9.760 & 68,2 & 32,5 & 9,4 & 1,5 & 2,8 & 230,7 & 23,5 & 5,9 & 72,2 \\
\hline Surinam & 458 & 74,6 & 29 & 9,9 & 0,6 & 2,4 & 8,9 & 19,5 & 6,9 & 70,2 \\
\hline $\begin{array}{l}\text { Trinidad y } \\
\text { Tobago }\end{array}$ & 1.333 & 12,9 & 21,4 & 11,1 & 0,4 & 1,6 & 19,8 & 14,8 & 8,1 & 69,8 \\
\hline Uruguay & 3.340 & 92,2 & 22,5 & 20,8 & 0,3 & 2,1 & 50,5 & 15,1 & 9,2 & 76,4 \\
\hline Venezuela & 27.657 & 94 & 30,3 & 8,8 & 1,7 & 2,5 & 597,8 & 21,4 & 5,1 & 73,7 \\
\hline
\end{tabular}

Fuentes: ๑ 2001-2007 Organización Panamericana de la Salud. Unidad de Análisis de Salud y Estadísticas. Cita sugerida: Organización Panamericana de la Salud, Unidad de Análisis de Salud y Estadísticas. Iniciativa Regional de Datos Básicos en Salud; Sistema de Información Técnica en Salud. Washington DC, 2007. http:// www.CLAP.ops-oms.org/web_2005/estadisticas

de educación y la mayor inversión en la salud pública con programas específicos para los sectores más vulnerables, son los factores comunes para los países con mejores resultados como sucede en Chile, Costa Rica y Cuba ( Schneider y cols, 2002; Astorga y cols, 2005; González y cols, 2006).

La OMS ha definido un mínimo de recursos que deben poseer las instalaciones de salud para ofrecer Cuidados Obstétricos Esenciales (Basic Essential Obstetric Care, EOC), necesarios para el manejo del embarazo, parto y puerperio normal y anormal no predecible (WHO y cols, 1997; WHO, 2000). Se definen dos niveles en el sistema de salud:

Básico o mínimo para el centro de salud primario. A. Manejo de patologías más comunes: anemia, diabetes, hipertensión (incluye manejo parenteral de antibióticos, ocitócicos, sedantes), los insumos deben ser considerados funcionales (reposición permanente). B. Manejo médico de emergencias obstétricas: hemorragias, sepsis, eclampsia. C. Procedimientos quirúrgicos menores: reparación de desgarros y manejo del alumbramiento.

Estándar óptimo de organización del sistema. Disponibilidad de traslado oportuno al primer centro de referencia con: A. Capacidad de parto monitorizado. B. Cuidados intensivos neonatales. C. Procedimientos quirúrgicos. D. Anestesia. E. Banco de sangre.

Los indicadores de salud en el área maternoinfantil han logrado un significativo avance, el resumen de la situación se observa en la Tabla VI (OPS, 2007). El riesgo de morir en la etapa perinatal esta en la región en niveles similares al promedio mundial: 17,3 por 1000 nacidos vivos (280.000 muertes/ año), la mayoría de ellas (55\%) en la primera semana de vida. El porcentaje de mejoría (estimaciones OMS/OPS) para la región entre el año 1990 y el 2005 es de 49 y $51 \%$ para la mortalidad infantil $(<1$ 
año) y la de menos de 5 años, respectivamente. La distribución de estos logros para los diferentes países es positivamente homogénea, con un rango de 7 a 64\%, con excepción de un país (Trinidad Tobago). Valores y tendencias catalogadas como aceptables a nivel global.

La atención profesional del parto para la región ha aumentado en un $24 \%$ desde el año 1990 al 2005 (desde 72 a $89 \%$ ). Aún permanecen regiones con población indígena en las cuales el $70 \%$ de los partos son atendidos por personal no capacitado. La mortalidad materna ha permanecido en niveles elevados, alcanzando según las fuentes referidas a un rango entre 72 a 140 por 100.000 nacidos vivos.

Tabla VI

MORTALIDAD INFANTIL Y MATERNA. CONTINENTE AMERICANO 2005-7

\begin{tabular}{|c|c|c|c|}
\hline & $\begin{array}{l}\text { Tasa de mortalidad } \\
\text { infantil (estimada por } \\
1.000 \text { nv). } 2007\end{array}$ & $\begin{array}{l}\text { Mortalidad de menores } \\
\text { de } 5 \text { años ( estimada por } \\
1.000 \mathrm{nv} \text { ). } 2006\end{array}$ & $\begin{array}{l}\text { Razón de mortalidad } \\
\text { materna reportada } \\
\text { (por } 100.000 \text { nv). } \\
2005-6\end{array}$ \\
\hline Argentina & 13,4 & 15,9 & 39,2 \\
\hline Bahamas & 13,8 & 17,9 & NA \\
\hline Bolivia & 45,6 & 62,9 & 230 \\
\hline Brasil & 23,6 & 30 & 76,1 \\
\hline Canadá & 4,8 & 5,9 & 5,9 \\
\hline Chile & 7,2 & 9 & 19,8 \\
\hline Colombia & 19,1 & 26,5 & 72,7 \\
\hline Costa Rica & 9,9 & 11,6 & 39,3 \\
\hline Cuba & 5,1 & 6,8 & 49,4 \\
\hline Ecuador & 21,1 & 26,5 & 85 \\
\hline El Salvador & 21,5 & 30,4 & 71,2 \\
\hline Estados Unidos de América & 6,3 & 7,9 & 13,1 \\
\hline Guadalupe & 6,8 & 9 & NA \\
\hline Guatemala & 30,1 & 41,2 & 148,8 \\
\hline Guyana & 42,9 & 59,4 & 161,2 \\
\hline Haití & 48,8 & 73,7 & 630 \\
\hline Honduras & 28,2 & 42,6 & 108 \\
\hline Jamaica & 13,6 & 17,5 & 95 \\
\hline Martinica & 6,6 & 8,4 & - \\
\hline México & 16,7 & 21,1 & 63,4 \\
\hline Nicaragua & 21,5 & 27 & 86,5 \\
\hline Panamá & 18,2 & 24,4 & 66 \\
\hline Paraguay & 32 & 39,2 & 153,5 \\
\hline Perú & 21,2 & 31,1 & 185 \\
\hline Puerto Rico & 7,2 & 8,9 & NA \\
\hline República Dominicana & 29,6 & 34,3 & 80 \\
\hline Surinam & 27,7 & 35,7 & NA \\
\hline Trinidad y Tobago & 12,4 & 18,2 & NA \\
\hline Uruguay & 13,1 & 16,4 & NA \\
\hline Venezuela & 17 & 22,3 & 59,9 \\
\hline
\end{tabular}

Fuentes: (c) 2001-2007 Organización Panamericana de la Salud. Unidad de Análisis de Salud y Estadísticas. Cita sugerida: Organización Panamericana de la Salud, Unidad de Análisis de Salud y Estadísticas. Iniciativa Regional de Datos Básicos en Salud; Sistema de Información Técnica en Salud. Washington DC, 2007. http://www.CLAP.ops-oms.org/ web_2005/estadisticas 
El porcentaje de mejoría para la región, en el indicador de razón de mortalidad materna entre el año 1990 - 2005 es de $28 \%$. La distribución de este indicador para los diferentes países es absolutamente heterogénea, los países con mejores indicadores presentan una tendencia hacia aún mayores mejorías proporcionales (Donoso, 2004a), en contrario, los países con situación de salud materna mas deteriorada presentan menores avances (Tabla VII).

\section{FUTURO}

Persisten problemas regionales importantes, principalmente la persistencia de indicadores in- aceptablemente elevados en poblaciones rurales, en etnias originarias, y aquellas con niveles menores de ingresos y educación.

La evaluación de los indicadores materno e infantil debe realizarse en forma conjunta. La visión de un continuo de cuidados, parece ser una apropiada estrategia para los países que debe incluir a los programas de salud publica de los gobiernos (Kerber y cols, 2007; WHO, 2005).

Existe consenso a nivel regional en las estrategias para mejorar la salud materno-infantil, entre las cuales destacan:

- aumentar la demanda y valoración social de los servicios de salud, que incluyan las demandas

Tabla VII

EVOLUCIÓN DE MORTALIDAD INFANTIL Y MATERNA. LATINO-AMÉRICA Y EL CARIBE

\begin{tabular}{|c|c|c|c|c|c|c|c|c|c|}
\hline \multirow[t]{2}{*}{ País } & \multicolumn{2}{|c|}{ MI } & \multirow[b]{2}{*}{ delta } & \multicolumn{2}{|c|}{$<5 a$} & \multicolumn{4}{|c|}{ MM } \\
\hline & 1990 & 2006 & & 1990 & 2006 & delta & 1990 & 2005 & delta \\
\hline Argentina & 25 & 14 & 44 & 29 & 16 & 45 & 100 & 77 & 23 \\
\hline Bahamas & 22 & 13 & 41 & 29 & 14 & 52 & 100 & 16 & 84 \\
\hline Bolivia & 89 & 50 & 44 & 125 & 61 & 51 & 650 & 290 & 55 \\
\hline Brasil & 48 & 19 & 60 & 57 & 20 & 65 & 220 & 110 & 50 \\
\hline Chile & 16 & 8 & 50 & 21 & 9 & 57 & 40 & 19 & 53 \\
\hline Colombia & 26 & 17 & 35 & 35 & 21 & 40 & 100 & 130 & -30 \\
\hline Costa Rica & 16 & 11 & 31 & 18 & 12 & 33 & 55 & 30 & 45 \\
\hline Cuba & 11 & 5 & 55 & 13 & 7 & 46 & 95 & 45 & 53 \\
\hline Ecuador & 43 & 21 & 51 & 57 & 24 & 58 & 150 & 210 & -40 \\
\hline El Salvador & 47 & 22 & 53 & 60 & 25 & 58 & 300 & 170 & 43 \\
\hline Guatemala & 60 & 31 & 48 & 82 & 41 & 50 & 200 & 290 & -45 \\
\hline Guyana & 64 & 46 & 28 & 88 & 62 & 30 & & 470 & \\
\hline Haití & 105 & 60 & 43 & 152 & 80 & 47 & 1000 & 670 & 33 \\
\hline Honduras & 45 & 23 & 49 & 58 & 27 & 53 & 220 & 280 & -27 \\
\hline Jamaica & 28 & 26 & 7 & 33 & 31 & 6 & 120 & 170 & -42 \\
\hline México & 42 & 29 & 31 & 53 & 35 & 34 & 110 & 60 & 45 \\
\hline Nicaragua & 52 & 29 & 44 & 68 & 36 & 47 & 160 & 170 & -6 \\
\hline Panamá & 27 & 18 & 33 & 34 & 23 & 32 & 55 & 130 & -136 \\
\hline Paraguay & 33 & 19 & 42 & 41 & 22 & 46 & 160 & 150 & 6 \\
\hline Perú & 58 & 21 & 64 & 78 & 25 & 68 & 280 & 240 & 14 \\
\hline República Dominicana & 50 & 25 & 50 & 65 & 29 & 55 & 110 & 150 & -36 \\
\hline Suriname & 35 & 29 & 17 & 48 & 39 & 19 & & 72 & \\
\hline Trinidad y Tabago & 30 & 33 & -10 & 34 & 38 & -12 & 90 & 45 & 50 \\
\hline Uruguay & 20 & 11 & 45 & 23 & 12 & 48 & 85 & 20 & 76 \\
\hline Venezuela & 27 & 18 & 33 & 33 & 21 & 36 & 120 & 57 & 53 \\
\hline América Latina y el Caribe & 43 & 22 & 49 & 55 & 27 & 51 & 180 & 130 & 28 \\
\hline
\end{tabular}

Fuentes: @ 2001-2007 Organización Panamericana de la Salud. Unidad de Análisis de Salud y Estadísticas. Cita sugerida: Organización Panamericana de la Salud, Unidad de Análisis de Salud y Estadísticas. Iniciativa Regional de Datos Básicos en Salud; Sistema de Información Técnica en Salud. Washington DC, 2007. Organización Mundial de la Salud. http://www.CLAP.ops-oms.org/web_2005/estadisticas. http://www.who.int/whosis/en/index.html 
originadas desde la comunidad.

- sistemas de salud eficientes en redes, de acuerdo a complejidad.

- cobertura y capacidad de recurso humano en atención de los controles antenatales, del parto, del recién nacido y del niño. Esto incluye los cuidados mínimos al momento del parto definidos por OMS (EOC), la evaluación pre y posconcepcional incluyendo la consejería en salud reproductiva.

- programas de registro y monitoreo adecuados.

- financiamiento orientado y sostenible en el tiempo hacia los sectores más vulnerables.

- alianzas entre los países y organizaciones internacionales, privilegiando las estrategias generadas desde la región.

- importancia de la vinculación de la salud materno-infantil con la mejoría de los derechos de la mujer en general.

Considerando los indicadores de salud materno-infantil como el reflejo de la salud y la calidad de vida de los países, es fundamental que su mejoría alcance a todas las personas, superando las inequidades existentes en la región.

\section{BIBLIOGRAFÍA *}

1. Acosta AA, Cabezas E, Chaparro JC. Present and future of maternal mortality in Latin America. Int J Gynaecol Obstet 2000;70:125-31.

2. Almeida-Filho N, Kawachi I, Filho AP, Dachs JN. Research on health inequalities in Latin America and the Caribbean: bibliometric analysis (1971-2000) and descriptive content analysis (1971-1995). Am J Public Health 2003;93:2037-43.

3. Astorga P, Berges A, Fitzgerald V. The standard of living in Latin America during the twentieth century. Economic History Review 2005;58:765-96.

4. Bahr J, Wehrhahn R. Life expectancy and infant mortality in Latin America: Soc Sci Med 1993;36:1373-82.

5. Barnes-Josiah D, Myntti C, Augustin A. The "three delays" as a framework for examining maternal mortality in Haiti. Soc Sci Med 1998;46:981-93.

6. Black RE, Morris SS, Bryce J. Where and why are 10 million children dying every year? Lancet 2003;361:2226-34.

7. Braveman $P$, Tarimo $E$. Social inequalities in health within countries: not only an issue for affluent nations: Soc Sci Med 2002;54:1621-35.

8. Bryce J, Daelmans B, Dwivedi A, Fauveau V, Lawn JE, Mason E, Newby H, Shankar A, Starrs A, Wardlaw T. Countdown to 2015 for maternal, newborn, and child survival: the 2008 report on tracking coverage of interventions. Lancet 2008;371:1247-58.

9. Bryce J, Requejo J. Tracking progress in maternal, newborn and child survival. The countdown 2008 re- port. UNICEF. 2008.

10. Casas JA, Dachs NW, Bambas A. Health disparities in Latin America and the Caribbean: the role of social and economic determinants. PAHO, 2001.

11. CEPAL. América Latina y el Caribe: el envejecimiento de la población: 1950-2050. Boletín Demográfico 2003, № 72. Hallado en: http://www.eclac.org/publicaciones/xml/1/13371/LCG2211.pdf

12. CEPAL. Panorama Social de América Latina 2007. Hallado en: http://www.cepal.org/publicaciones/ xml/5/30305/PSE2007_VersionCompleta.pdf

13. Donoso E. Reducción de la mortalidad materna en Chile de 1990 a 2000. Rev Panam Salud Publica 2004a;15:326-30.

14. Donoso E. Inequalities in infant mortality in Santiago. Rev Med Chil 2004b;132:461-6.

15. Donoso E. ¿Qué esta pasando con la mortalidad materna en Chile en los inicios del siglo XXI? Rev Chil Obstet Ginecol 2006;71:157-8.

16. Donoso E, Carvajal JA. Maternal, perinatal and infant outcome of spontaneous pregnancy in the sixth decade of life. Maturitas 2008;59:381-6.

17. Donoso E, Villarroel L. Reproductive risk of women over 40 years old. Rev Med Chil 2003;131:55-9.

18. González R, Merialdi M, Lincetto O, Lauer J, Becerra C, Castro R, García P, Saugstad OD, Villar J. Reduction in neonatal mortality in Chile between 1990 and 2000. Pediatrics 2006;117:e949-54.

19. Jiménez J, Romero MI. Reducing infant mortality in Chile: success in two phases. Health Aff (Millwood) 2007;26:458-65.

20. Kerber KJ, Graft-Johnson JE, Bhutta ZA, Okong P, Starrs A, Lawn JE. Continuum of care for maternal, newborn, and child health: from slogan to service delivery. Lancet 2007;370:1358-69.

21. Khan KS, Wojdyla D, Say L, Gulmezoglu AM, Van Look PF. WHO analysis of causes of maternal death: a systematic review: Lancet 2006;367:1066-74.

22. Lawn JE, Cousens S, Zupan J., 4 million neonatal deaths: When? Where? Why? Lancet 2005;365:891-900.

23. Mathers CD, Stein C, Fat DM. Global Burden of Disease 2000: version2, methods and results. World Health Organization, 2000.

24. OPS. Plan de acción regional para la reducción de la mortalidad materna en las Américas. Bol Oficina Sanit Panam 1991;110:448-54.

25. OPS. Salud en Las Américas, 2007. Publicación Científica y Técnica № 622. Panamerican Health Organization. Washington DC, 2007.

26. Schneider MC, Castillo-Salgado C, Loyola-Elizondo E, Bacallao J, Mujica OJ, Vidaurre M, Alleyne GA. Trends in infant mortality inequalities in the Americas: 19551995. J Epidemiol Community Healt 2002;56:538-41.

27. United Nations. Declaración del Milenio. 2000. Hallado en: http://www.un.org./millennium/declaration/ares552e. 
htm

28. United Nations. Department of Economic and Social Affaire, Population Division. World Population Prospects: The 2004 Revision. Population database. Hallado en: http://esa.un.org/unpp/index.asp.panel6

29. United Nations. World Urbanization Prospects: The 2005 Revision. United Nations Department of Economic and Social Affairs/Population Division. 2005.

30. Victora CG, Barros FC, Huttly SR, Teixeira AM, Vaughan JP. Early childhood mortality in a Brazilian cohort: the roles of birthweight and socioeconomic status. Int J Epidemiol 1992;21:911-5

31. Wagstaff A, Bustreo F, Bryce J, Claeson M. Child health: reaching the poor. Am $\mathrm{J}$ Public Health 2004;94:726-36.

32. WHO. Essential obstetrics care. Fact scheet № 245 ; 2000. Hallado en: www.who.int/entity/mediacentre/ factsheets/fs245/en/index.html

33. WHO. Make every mother and child count. World
Health Report 2005.

34. WHO. Making a difference in countries. World Health Organization 2006a. WHO Press.

35. WHO. Neonatal and Perinatal Mortality. Country, regional and global estimates. World Health Organization. 2006b. WHO Press.

36. WHO. WHO Statistical Information System (WHOSIS), 2008. Hallado en: http://www.who.int/whosis/en/ index.html

37. WHO, UNICEF, UNFPA. Guidelines for monitoring the availability and use of obstetric services. New York, 1997.

38. WHO, UNICEF, UNFPA. Maternal mortality in 2005: estimates developed by WHO, UNICEF, UNFPA and The World Bank. 2007.

* Todos los sitios web fueron visitados al 31 de Agosto de 2008. 